\title{
A behavioral approach to estimation in the presence of disturbances
}

\author{
Ricardo Pereira, Paula Rocha, Lorenzo Ntogramatzidis
}

\begin{abstract}
In this paper we study the problem of estimation in the presence of disturbances within the context of the behavioral approach developed by J.C. Willems. For this purpose, we use the behavioral theory of observers introduced by Valcher, Willems, Trentelman and Trumpf, combined with the notions of behavioral invariance, conditioned invariance and behavioral detectability subspaces. With these tools we provide necessary and sufficient conditions for the solvability of the aforementioned problem together with the construction of an estimator.
\end{abstract}

Index Terms-Behavior, invariance, observer, conditioned invariance, detectability, estimation, disturbance.

\section{INTRODUCTION}

Conditioned invariance is one of the cornerstones of the so-called geometric approach to control theory. It was introduced in [1] as the dual of controlled invariance. The modern point of view of defining conditioned invariant subspaces in terms of the existence of a certain class of observers of linear combinations of the state vector originates from [2] and [3]. A stability characterization of conditioned invariance leads to the notion of detectability subspaces. A detectability subspace is related to the existence of an asymptotic observer which reconstructs the state vector modulo its components on that subspace. This system-theoretic point of view is the one adopted in the monograph [4]. Conditioned invariance and detectability subspaces are the fundamental building blocks in the geometric solution of the so-called unknown-input observation problem, whose relevance is very well established in contexts such as fault detection and isolation and non-interacting control [5]. In broad lines, the unknown-input observation problem can be formulated as follows: given a system with state dynamics driven by a disturbance $d$, a measurable output $y$ and a non-measurable output $z$ (which are both linear functions of the state), one wishes to construct (if possible) another system that produces an estimate $\hat{z}$ of the non-measurable output based on the measurement $y$. The answer to this problem can be given in terms of detectability subspaces [4], [6], [7], [8].

The aim of this paper is to study this problem in the more general framework of behavioral systems, [9]. The behavioral approach has proved to be a valuable tool in systems theory because, being more general, it offers additional insight and new perspectives into concepts and notions that are typically only dealt with by classical methods. This is in particular the case of conditioned invariance and detectability subspaces and their link with the existence of observers. This aspect is not surprising in light of previous contributions on observability in a behavioral framework, see [10].

Ricardo Pereira is with the CIDMA - Center for Research and Development in Mathematics and Applications, Department of Mathematics, University of Aveiro, Portugal. E-mail: ricardopereira@ua.pt.

Paula Rocha is with the SYSTEC, Faculty of Engineering, University of Porto, Portugal. E-mail: mprocha@fe.up.pt.

L. Ntogramatzidis is with the School of Electrical Engineering, Computing and Mathematical Sciences, Curtin University, Perth, Australia. E-mail: L.Ntogramatzidis@curtin.edu.au.

This work is supported by The Center for Research and Development in Mathematics and Applications (CIDMA) through the Portuguese Foundation for Science and Technology, references UIDB/04106/2020 and UIDP/04106/2020. Moreover, it was also partially supported by UIDB/00147/2020 - SYSTEC - Research Center for Systems and Technologies funded by national funds through the FCT/MCTES (PIDDAC) and by the Australian Research Council under the grant DP190102478.
In this approach, the most relevant feature of a system is the set of (possibly multivariate) signals that satisfy its laws, known as the system behavior. As a starting point, the components of the signals (system variables) are not divided into inputs and outputs, but may be split in several ways according to the problems under consideration. Here, given our focus on estimation, we assume that the system variables are divided into measured variables, to-be-estimated variables and, possibly, disturbances (which play the role of unknown inputs in the classical approach). To investigate the behavioral estimation problem, we rely on the behavioral theory of observers introduced in [11] and [10], as well as on our previous results on conditioned invariant and behavioral detectability subspaces presented in [12]. Roughly speaking, an observer for a given behavior $\mathscr{B}$ is a second behavior $\widehat{\mathscr{B}}$ that shares the measured variables with $\mathscr{B}$ and produces a suitable estimate of the to-beestimated variables, in the sense that the error behavior (i.e., the set of all estimation error signals) has certain desired properties, see Figure 1:

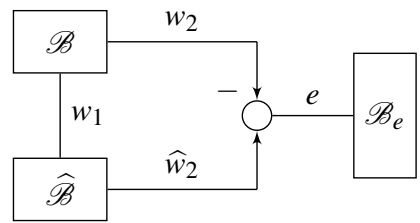

Figure 1: Behavioral observation interconnection.

On the other hand, conditioned invariant and detectability subspaces are behaviors $\mathscr{V}_{e}$ contained in the error behavior $\mathscr{B}_{e}$ of an observer, such that the quotient behavior $\mathscr{B}_{e} / \mathscr{V}_{e}$ has suitable properties: specifically, autonomy and stability. Thus, loosely speaking, $\mathscr{V}_{e}$ is a conditioned invariant subspace if $\mathscr{B}_{e}$ is autonomous modulo $\mathscr{V}_{e}$, and it is a detectability subspace if $\mathscr{B}_{e}$ is stable modulo $\mathscr{V}_{e}$. All these concepts are reviewed in the main body of this paper.

The definition of conditioned invariance given in this paper differs from the one we first gave in [13]. Indeed, our first definition was based on an inadequate notion of behavioral invariance, which implied that the sole existence of an observer was sufficient to guarantee conditioned invariance. This problem was later solved in [12] by adjusting the definition of behavioral invariance.

The main contribution of this paper is the formulation and (whenever possible) the solution of two behavioral estimation problems: the first one without disturbances, and the second one involving disturbances. The existence of solutions for these problems is expressed in terms of the existence of suitable behavioral detectability subspaces.

We start by reviewing the relevant notions on behaviors and on the behavioral theory of observers (Section 2). In Section 3 we introduce invariant, conditioned invariant, and behavioral detectability subspaces. In Sections 4 and 5 we study the behavioral estimation problem with and without disturbances, respectively. Concluding remarks are given in Section 6.

\section{PREliminaries - BeHAVIORS AND BeHAVIORAL OBSERVERS}

\section{A. Behaviors}

Behavioral systems theory is based on the idea that a system is characterized by its behavior - the set of all the signals in a 
given signal universe that are admissible according to the laws of the phenomenon described by the system.

In this paper we consider behaviors $\mathscr{B}$ that are linear subspaces of the universe $\mathscr{U}=\mathscr{C}^{\infty}\left(\mathbb{R}, \mathbb{R}^{\mathrm{w}}\right)$, for some $\mathrm{w} \in \mathbb{N}$, consisting of the solutions of systems of linear, homogeneous differential equations with constant coefficients in w variables. Specifically,

$$
\mathscr{B}=\left\{w \in \mathscr{C}^{\infty}\left(\mathbb{R}, \mathbb{R}^{\mathrm{w}}\right): R\left(\frac{d}{d t}\right) w=0\right\},
$$

where $R(s)$ is a suitably sized matrix with entries in the ring $\mathbb{R}[s]$ of polynomials in $s$. Noting that $\mathscr{B}$ can be regarded as the kernel of a linear map $R\left(\frac{d}{d t}\right): \mathscr{C}^{\infty}\left(\mathbb{R}, \mathbb{R}^{\mathrm{w}}\right) \rightarrow \mathscr{C}^{\infty}\left(\mathbb{R}, \mathbb{R}^{\mathrm{g}}\right)$, for some $g \in \mathbb{N}$, we write $\mathscr{B}=\operatorname{ker} R\left(\frac{d}{d t}\right)$. Whenever the context is clear, we will omit the indeterminate $s$ and the operator $\frac{d}{d t}$. We next recall some basic properties of behaviors that will be useful in the sequel, [14], [15].

Whereas an operator $R\left(\frac{d}{d t}\right)$ uniquely defines a behavior, the same behavior can be described as the kernel of different operators. In particular, given any unimodular matrix $U$, we have $\operatorname{ker} R=\operatorname{ker} U R$. Moreover, $\mathscr{B}_{1}=\operatorname{ker} R_{1} \subset \mathscr{B}_{2}=\operatorname{ker} R_{2}$ if and only if there exists a polynomial matrix $\bar{R}$ such that $R_{2}=\bar{R} R_{1}$. Furthermore, since every polynomial matrix $R$ can be brought to the form $\left[\begin{array}{l}F \\ 0\end{array}\right]$ where $F$ is a full row rank polynomial matrix, by pre-multiplication by a suitable unimodular matrix $U$, we obtain

$$
\operatorname{ker} R=\operatorname{ker} U R=\operatorname{ker}\left[\begin{array}{l}
F \\
0
\end{array}\right]=\operatorname{ker} F .
$$

Hence every behavior can be regarded as the kernel of an operator associated to a full row rank polynomial matrix. Unless stated otherwise, we shall assume that this is the case.

Quotients of behaviors play an important role in the behavioral theory. In [15, Thm. 2.56] it is shown that if $\mathscr{B}_{1}$ is a sub-behavior of $\mathscr{B}_{2}$, the quotient of the two behaviors $\mathscr{B}_{2} / \mathscr{B}_{1}$ (defined as the quotient of $\mathscr{B}_{2}$ by $\mathscr{B}_{1}$ as vector spaces) also admits the structure of a behavior. Indeed, if $\mathscr{B}_{1}=\operatorname{ker} R_{1}$ and $\mathscr{B}_{2}=\operatorname{ker} \bar{R} R_{1}$, with $R_{1}$ full row rank (w.l.o.g.), as mentioned in [16, Lemma 2.13], the quotient behavior $\mathscr{B}_{2} / \mathscr{B}_{1}$ is isomorphic to $\operatorname{ker} \bar{R}$. For the definition of isomorphism of behaviors, we refer the reader to [17, Sect. 2.5].

Given a behavior $\mathscr{B}$ consisting of vector signals $w$, it is often useful to analyse its degrees of freedom. Typically, there will be a set of components of $w$ (system variables) which are free, and a complementary set of variables which are determined by the free variables, together with initial conditions. However, in some cases, no component of $w$ is completely free. This can be alternatively expressed by saying that two signals that coincide in the past must also coincide in the future, i.e., the past completely determines the future. In such case, we say that the behavior $\mathscr{B}$ is autonomous. Autonomous behaviors are crucial in this paper. The formal definition given next takes into account that we are dealing with linear systems, and considers what happens to a signal which is zero (rather than with two signals which coincide) in the past.

Definition 1: A behavior $\mathscr{B}$ is autonomous if for every $w \in \mathscr{B}$ we have that $w(t)=0$ for all $t \leq 0$ implies $w \equiv 0$.

Autonomy can be characterized in terms of the polynomial operators that describe a behavior, as stated in the following lemma.

Lemma 1: A behavior $\mathscr{B}=\operatorname{ker} R$ is autonomous if and only if $R$ has full column rank over $\mathbb{R}[s]$.

Besides autonomy, another relevant property is stability; the definition and necessary and sufficient condition for stability stated below can be found in [14, Section 7].

Definition 2: A behavior $\mathscr{B}$ is said to be stable if, for every $w \in \mathscr{B}$, $\lim _{t \rightarrow+\infty} w(t)=0$.

Lemma 2: A behavior $\mathscr{B}=\operatorname{ker} R$ is stable if and only if $R(\lambda)$ has full column rank for all $\lambda \in \mathbb{C}_{0}^{+}:=\{\lambda \in \mathbb{C}: \operatorname{Re}(\lambda) \geq 0\}$.
In particular, stable behaviors are autonomous, as the free components of $w$ do not necessarily converge to zero as $t$ tends to infinity.

\section{B. Behavioral observers}

As is well-known, in the classical state space approach, an observer of a linear time-invariant state-space system $\Sigma$ is a system $\Omega$ that produces an estimate $\hat{x}$ of the state $x$ on the basis of the known values of the input $u$ and the measured values of the output $y$. Observers may be classified according to the properties of the corresponding estimation error $e=\widehat{x}-x$. In particular, $\Omega$ is said to be:

- a tracking state observer for $\Sigma$ if, for any pair of initial values $(x(0), \widehat{x}(0)), e(0)=\widehat{x}(0)-x(0)$ is such that:

$$
e(0)=0 \Rightarrow[e(t)=0, \forall t \geq 0] ;
$$

- an asymptotic state observer for $\Sigma$ if it is a tracking state observer, and $\lim _{t \rightarrow+\infty} e(t)=0$ for every initial condition $e(0)$,

see e.g. [4]. In order to study observers in the behavioral framework, one has to consider linear time-invariant differential system with behavior $\mathscr{B}_{\left(w_{1}, w_{2}\right)}$, where the system variable $w=\left(w_{1}, w_{2}\right)$ is partitioned into measured variables $w_{1}$ and to-be-estimated variables $w_{2}$, with $w_{1}$ and $w_{2}$ components, respectively ${ }^{1}$.

In [11], [10], the behavioral notions of observer and tracking/asymptotic observer of $w_{2}$ from $w_{1}$ for $\mathscr{B}_{\left(w_{1}, w_{2}\right)}$ are as follows.

Definition 3: Given a linear time-invariant differential behavior $\mathscr{B}_{\left(w_{1}, w_{2}\right)}$, let $\widehat{\mathscr{\mathscr { B }}}_{\left(w_{1}, \widehat{w}_{2}\right)}$ be a behavior such that the universe $\mathscr{U}_{\left(w_{1}, \widehat{w}_{2}\right)}$ coincides with the universe $\mathscr{U}_{\left(w_{1}, w_{2}\right)}=\mathscr{C}^{\infty}\left(\mathbb{R}, \mathbb{R}^{w_{1}} \times \mathbb{R}^{w_{2}}\right)$ of the variable $\left(w_{1}, w_{2}\right) . \widehat{\mathscr{B}}_{\left(w_{1}, \widehat{w}_{2}\right)}$ is said to be an observer of $w_{2}$ from $w_{1}$ (for $\mathscr{B}_{\left(w_{1}, w_{2}\right)}$ ) if $\widehat{w}_{2}$ is to be understood as an estimate of $w_{2}$. Moreover, defining the error of the estimate by $e=\widehat{w}_{2}-w_{2}$ and the corresponding error behavior ${ }^{2}$

$$
\begin{aligned}
\mathscr{B}_{e}= & \left\{e=\widehat{w}_{2}-w_{2}: \exists w_{1}\right. \text { s.t. } \\
& \left.\left(w_{1}, w_{2}\right) \in \mathscr{B}_{\left(w_{1}, w_{2}\right)},\left(w_{1}, \widehat{w}_{2}\right) \in \widehat{\mathscr{B}}_{\left(w_{1}, \widehat{w}_{2}\right)}\right\} .
\end{aligned}
$$

$\widehat{\mathscr{B}}_{\left(w_{1}, \widehat{w}_{2}\right)}$ is said to be:

- a tracking observer of $w_{2}$ from $w_{1}$ if, whenever $\left(w_{1}, w_{2}\right) \in$ $\mathscr{B}_{\left(w_{1}, w_{2}\right)}$ and $\left(w_{1}, \widehat{w}_{2}\right) \in \widehat{\mathscr{B}}_{\left(w_{1}, \widehat{w}_{2}\right)}$ with $\widehat{w}_{2}(t)=w_{2}(t)$ for $t \in$ $(-\infty, 0]$, then $\widehat{w}_{2}(t)=w_{2}(t), \forall t \in \mathbb{R}$. In other words

$$
\left[e_{\mid(-\infty, 0]} \equiv 0, e \in \mathscr{B}_{e}\right] \Rightarrow[e(t)=0, \forall t \in \mathbb{R}],
$$

i.e., $\mathscr{B}_{e}$ is autonomous.

- an asymptotic observer of $w_{2}$ from $w_{1}$ if it is a tracking observer and $\lim _{t \rightarrow+\infty} e(t)=0$, for all $e \in \mathscr{B}_{e}$, i.e, $\mathscr{B}_{e}$ is stable.

Remark 1: Throughout this note we shall assume that an observer $\widehat{\mathscr{B}}_{\left(w_{1}, \widehat{w}_{2}\right)}$ of $w_{2}$ from $w_{1}$ for a behavior $\mathscr{B}_{\left(w_{1}, w_{2}\right)}$ is such that the $w_{1}$ behavior of $\mathscr{B}_{\left(w_{1}, w_{2}\right)}$ is contained in the $w_{1}$-behavior of $\widehat{\mathscr{B}}_{\left(w_{1}, \widehat{w}_{2}\right)}$. Thus, the observer "accepts" all the $w_{1}$-trajectories coming from the original behavior. Using the terminology of [11], this means that $\widehat{\mathscr{B}}_{\left(w_{1}, \widehat{w}_{2}\right)}$ should be an acceptor of $w_{1}$ for $\mathscr{B}_{\left(w_{1}, w_{2}\right)}$. With the terminology of [10], this means that $\widehat{\mathscr{B}}_{\left(w_{1}, \widehat{w}_{2}\right)}$ should be nonintrusive.

In case a tracking observer of $w_{2}$ from $w_{1}$ for $\mathscr{B}_{\left(w_{1}, w_{2}\right)}$ exists, we shall say that $w_{2}$ is trackable from $w_{1}$ in $\mathscr{B}_{\left(w_{1}, w_{2}\right)}$. In [11], the following test is given for trackability.

${ }^{1}$ From now on, when considering a behavior, we shall indicate the corresponding system variables as subscript. Moreover, whenever two behaviors have different system variables but share the same universe, we sometimes ignore the difference of the system variables and allow ourselves to compare them in terms of inclusion or equality.

${ }^{2}$ The fact that $\mathscr{B}_{e}$ is a behavior follows from the variable elimination principle in [15, Cor. 2.38]. 
Proposition 1: Let $\mathscr{B}_{\left(w_{1}, w_{2}\right)}$ be described by

$$
R_{2}\left(\frac{d}{d t}\right) w_{2}=R_{1}\left(\frac{d}{d t}\right) w_{1}
$$

with $R_{1} \in \mathbb{R}^{g \times w_{1}}[s]$ and $R_{2} \in \mathbb{R}^{g \times w_{2}}[s]$. Then, $w_{2}$ is trackable from $w_{1}$ for $\mathscr{B}_{\left(w_{1}, w_{2}\right)}$ if and only if $R_{2}$ has full column rank.

A trivial observer for $\mathscr{B}_{\left(w_{1}, w_{2}\right)}$ defined in this proposition is the behavior $\widehat{\mathscr{B}}_{\left(w_{1}, \widehat{w}_{2}\right)}$ described by

$$
R_{2}\left(\frac{d}{d t}\right) \widehat{w}_{2}=R_{1}\left(\frac{d}{d t}\right) w_{1} .
$$

The error behavior corresponding to this observer is $\mathscr{B}_{e}=\operatorname{ker} R_{2}$, which coincides with the hidden behavior of $w_{2}$ defined in [10] as

$$
\mathscr{N}_{w_{2}}\left(\mathscr{B}_{\left(w_{1}, w_{2}\right)}\right)=\left\{w_{2} \mid\left(0, w_{2}\right) \in \mathscr{B}_{\left(w_{1}, w_{2}\right)}\right\} .
$$

Hence, the hidden behavior consists of all the non-measured signals $w_{2}$ that are compatible with the zero measurement $w_{1}=0$. Note that this is in fact nothing else than the behavior of the $w_{2}$-variable that is unobservable from $w_{1}$; we refer the reader to [9, Sect. 4.3.2] for a definition of observability in the behavioral context.

Thus, Proposition 1 shows that trackability is equivalent to the autonomy of the hidden behavior, cf [10, Def. 4.1 and Prop. 4.4].

In the case where an asymptotic observer of $w_{2}$ from $w_{1}$ for $\mathscr{B}_{\left(w_{1}, w_{2}\right)}$ exists, we say that $w_{2}$ is detectable from $w_{1}$ in $\mathscr{B}_{\left(w_{1}, w_{2}\right)}$. It is easy to see that if the hidden behavior $\operatorname{ker} R_{2}$ is stable, the trivial observer (1) is an asymptotic observer. On the other hand, it is shown in [10, Def. 4.1 and Prop. 4.4] that the converse is also true. Thus, detectability is equivalent to the stability of the hidden behavior.

\section{INVARIANCE, CONDITIONED INVARIANCE AND DETECTABILITY SUBSPACES}

The aim of this section is to introduce the behavioral notions of conditioned invariant and detectability subspaces, which play an important role in the solution of the behavioral estimation problem.

\section{A. Invariant sub-behaviors}

Given an autonomous state space system

$$
\frac{d}{d t} x=A x,
$$

with state space $\mathscr{X}$, a subspace $\mathscr{V}_{\mathscr{X}}$ of $\mathscr{X}$ is said to be invariant with respect to the system dynamics, or simply A-invariant, if all state signals that start in $\mathscr{V}_{\mathscr{X}}$ remain in this subspace [4].

In the behavioral approach we consider the invariance of a given sub-behavior $\mathscr{V}$ under the dynamics associated to a (non-necessarily autonomous) behavior $\mathscr{B}$, defined as follows [18].

Definition 4: Let $\mathscr{B}_{w}$ be a behavior. A behavior $\mathscr{V}_{w}$ is said to be $\mathscr{B}_{w}$-invariant if the following condition holds:

$$
\mathscr{V}_{w} \subset \mathscr{B}_{w} \text { and }\left[w_{\mid(-\infty, 0]} \in \mathscr{V}_{w_{\mid(-\infty, 0]}}, w \in \mathscr{B}_{w}\right] \Rightarrow\left[w \in \mathscr{V}_{w}\right],
$$

where, as usual, $w_{\mid(-\infty, 0]}$ denotes the restriction of $w$ to the interval $(-\infty, 0]$ and $\mathscr{V}_{w_{\mid(-\infty, 0]}}$ is the set of restrictions of the signals $v \in \mathscr{V}_{w}$ to the same interval.

This means that the signals of $\mathscr{B}_{w}$ whose past is compatible with $\mathscr{V}_{w}$ must remain in $\mathscr{V}_{w}$ in the future, i.e., they in fact belong to $\mathscr{V}_{w}$. Thus, loosely speaking, $\mathscr{B}_{w}$ is autonomous "up to" $\mathscr{V}_{w}$. This is stated in the following characterization of invariance given in [18, Thm. 7].

Proposition 2: Let $\mathscr{B}_{w}$ and $\mathscr{V}_{w} \subset \mathscr{B}_{w}$ be two behaviors. Then, $\mathscr{V}_{w}$ is $\mathscr{B}_{w}$-invariant if and only if $\mathscr{B}_{w} / \mathscr{V}_{w}$ is autonomous.

Recalling what was said in sub-section II-A on quotients of behaviors, the foregoing proposition leads to the following characterization of invariance in terms of matrix representations.

Proposition 3: Given two behaviors $\mathscr{V}_{w}=\operatorname{ker} V$ and $\mathscr{B}_{w}=\operatorname{ker} \bar{R} V$, with $V$ full row rank, then $\mathscr{V}_{w}$ is $\mathscr{B}_{w}$-invariant if and only if the matrix $\bar{R}$ is full column rank.

\section{B. Conditioned invariance}

We now introduce behavioral conditioned invariance based on the definitions of behavioral invariance (cf Definition 4) and behavioral observer (cf Definition 3). The state-space notion of conditioned invariance rests on the concept of observer modulo a subspace of the state space, see [4]. Indeed, consider a system with state space $\mathscr{X}$ and state $x$. A subspace $\mathscr{V}_{\mathscr{X}}$ of $\mathscr{X}$ is said to be conditioned invariant if there exists a system $\Omega$ with state-space $\mathscr{X}$ and state $\widehat{x}$ which is a tracking observer for $\mathscr{X} / \mathscr{V}_{\mathscr{X}}$ in the following sense: if $e(0)=\widehat{x}(0)-x(0) \in \mathscr{V}_{\mathscr{X}}$, then $e(t)=\widehat{x}(t)-x(t) \in \mathscr{V}_{\mathscr{X}}$ for all $t \geq 0$.

With this in mind, and taking into account the behavioral definition of observer (Definition 3), we now introduce a behavioral definition of tracking observer modulo $\mathscr{V}_{e}$.

In the sequel, whenever the error is defined as in Definition 3, i.e., $e=\widehat{w}_{2}-w_{2}$, the universes $\mathscr{U}_{w_{2}}, \mathscr{U}_{\widehat{w}_{2}}$ and $\mathscr{U}_{e}$ coincide.

Definition 5: Let $\mathscr{B}_{\left(w_{1}, w_{2}\right)}$ and $\widehat{\mathscr{B}}_{\left(w_{1}, \widehat{w}_{2}\right)}$ be two linear time-invariant differential behaviors. Define the error behavior $\mathscr{B}_{e}$ as in Definition 3, and let $\mathscr{V}_{e}$ be a sub-behavior of $\mathscr{B}_{e}$. The behavior $\widehat{\mathscr{B}}_{\left(w_{1}, \widehat{w}_{2}\right)}$ is said to be a tracking observer of $w_{2}$ from $w_{1}$ modulo $\mathscr{V}_{e}$ if

$$
\left[\begin{array}{l}
e_{\mid(-\infty, 0]} \in\left(\mathscr{V}_{e}\right)_{\mid(-\infty, 0]} \\
e \in \mathscr{B}_{e}
\end{array}\right] \Rightarrow\left[e \in \mathscr{V}_{e}\right] .
$$

Note that this means that $\mathscr{V}_{e}$ is $\mathscr{B}_{e}$-invariant. Thus Proposition 2 leads to the next lemma.

Lemma 3: The behavior $\widehat{\mathscr{B}}_{\left(w_{1}, \widehat{w}_{2}\right)}$ is a tracking observer of $w_{2}$ from $w_{1}$ modulo $\mathscr{V}_{e}$ if and only if $\mathscr{B}_{e} / \mathscr{V}_{e}$ is autonomous.

As in the state space case, in the behavioral context we define the conditioned invariance of a behavior $\mathscr{V}_{e}$ in terms of existence of a behavioral tracking observer modulo $\mathscr{V}_{e}$.

Definition 6: Let $\mathscr{B}_{\left(w_{1}, w_{2}\right)}$ be a linear time-invariant differential behavior with measured variable $w_{1}$, and to-be-estimated variable $w_{2}$ in a universe $\mathscr{U}_{w_{2}}$. A behavior $\mathscr{V}_{e} \subset \mathscr{U}_{e}=\mathscr{U}_{w_{2}}$ is said to be conditioned invariant if there exists a (behavioral) tracking observer of $w_{2}$ from $w_{1}$ modulo $\mathscr{V}_{e}$.

Example 3.1: Consider the behavior $\mathscr{B}_{\left(w_{1}, w_{2}\right)}$ described by $R_{2}\left(\frac{d}{d t}\right) w_{2}=R_{1}\left(\frac{d}{d t}\right) w_{1}$ with

$$
R_{2}\left(\frac{d}{d t}\right)=\left(\frac{d^{2}}{d t^{2}}+4\right)\left[\frac{d}{d t}+1 \quad \frac{d}{d t}+5\right] \text { and } R_{1}=\left(\frac{d^{2}}{d t^{2}}+8\right) .
$$

Because $R_{2}$ has not full column rank, then $w_{2}$ is not trackable from $w_{1}$ in $\mathscr{B}_{\left(w_{1}, w_{2}\right)}$. Considering the trivial observer $\widehat{\mathscr{B}}_{\left(w_{1}, \widehat{w}_{2}\right)}$ described by $R_{2}\left(\frac{d}{d t}\right) \widehat{w}_{2}=R_{1}\left(\frac{d}{d t}\right) w_{1}$, the error behavior is $\mathscr{B}_{e}=\operatorname{ker} R_{2}$. Let $\mathscr{V}_{e}$ be the sub-behavior of $\mathscr{B}_{e}$ given by $\mathscr{V}_{e}=\operatorname{ker}\left[\begin{array}{ll}\frac{d}{d t}+1 & \frac{d}{d t}+5\end{array}\right]$. Since $\mathscr{B}_{e} / \mathscr{V}_{e} \simeq \operatorname{ker}\left(\frac{d^{2}}{d t^{2}}+4\right)$ is autonomous, then $\mathscr{V}_{e}$ is $\mathscr{B}_{e}$-invariant which implies by definition that $\mathscr{V}_{e}$ is a conditioned invariant behavior. $\diamond$

Since conditioned invariance is related to the existence of behavioral observers whose error behaviors have specific properties, knowing which error behaviors can be obtained by designing a suitable observer becomes a central issue. Such error behaviors are called achievable, [10], and are defined and characterized as follows.

Definition 7: Let $\mathscr{B}_{\left(w_{1}, w_{2}\right)}$ be a linear time-invariant differential behavior with observed variable $w_{1}$, and to-be-estimated variable $w_{2}$ in a universe $\mathscr{U}_{w_{2}}$. A behavior $\mathscr{E}_{e} \subset \mathscr{U}_{e}=\mathscr{U}_{w_{2}}$ is said to be an achievable error behavior if there exists an observer $\widehat{\mathscr{B}}_{\left(w_{1}, \widehat{w}_{2}\right)}$ of $w_{2}$ from $w_{1}$ with error behavior $\mathscr{B}_{e}$ such that $\mathscr{E}_{e}=\mathscr{B}_{e}$.

Proposition 4: [10, Prop. 3.5] Let $\mathscr{B}_{\left(w_{1}, w_{2}\right)}$ be a linear timeinvariant differential behavior with observed variable $w_{1}$, and to-beestimated variable $w_{2}$ in a universe $\mathscr{U}_{w_{2}}$. Then the behavior $\mathscr{E}_{e} \subset$ $\mathscr{U}_{e}=\mathscr{U}_{w_{2}}$ is an achievable error behavior if and only if the hidden behavior $\mathscr{N}_{w_{2}}\left(\mathscr{B}_{\left(w_{1}, w_{2}\right)}\right)$ satisfies the condition $\mathscr{N}_{w_{2}}\left(\mathscr{B}_{\left(w_{1}, w_{2}\right)}\right) \subset \mathscr{E}_{e}$.

Now, we can say that $\mathscr{V}_{e}$ is conditioned invariant if and only if it is $\mathscr{E}_{e}$-invariant, for some achievable error behavior $\mathscr{E}_{e}$. By Proposition 4 this leads to the following result [13]. 
Corollary 1: Let $\mathscr{B}_{\left(w_{1}, w_{2}\right)}$ be a behavior with observed variable $w_{1}$, and to-be-estimated variable $w_{2}$ in a universe $\mathscr{U}_{w_{2}}$. A behavior $\mathscr{V}_{e} \subset \mathscr{U}_{e}=\mathscr{U}_{w_{2}}$ is conditioned invariant if and only if there exists a behavior $\mathscr{E}_{e} \supset \mathscr{N}_{w_{2}}\left(\mathscr{B}_{\left(w_{1}, w_{2}\right)}\right)$ such that $\mathscr{V}_{e}$ is $\mathscr{E}_{e}$-invariant.

A full characterization of conditioned invariance in terms of the polynomial matrices associated to the relevant behaviors is given in the next proposition, [12].

Proposition 5: Let $\mathscr{B}_{\left(w_{1}, w_{2}\right)}$ be a behavior with hidden behavior $\mathscr{N}_{w_{2}}\left(\mathscr{B}_{\left(w_{1}, w_{2}\right)}\right)=\operatorname{ker} R_{2}$. Then a behavior $\mathscr{V}_{e} \subset \mathscr{U}_{e}=\mathscr{U}_{w_{2}}$ described by $\mathscr{V}_{e}=\operatorname{ker} V$ is conditioned invariant if and only if $\exists q \in \mathbb{R}[s], q \neq 0$, such that $\operatorname{ker} R_{2} \subset \operatorname{ker} q V$.

The proof is omitted because it can be obtained as a particular case of the proof of Proposition 6.

Remark 2: The existence of a polynomial $q \in \mathbb{R}[s], q \neq 0$, such that $\operatorname{ker} R_{2} \subset \operatorname{ker} q V$ mentioned above is equivalent to the (easily checkable) existence of a rational matrix $G(s)$ such that $V=G R_{2}$. Indeed, $\operatorname{ker} R_{2} \subset \operatorname{ker} q V$ implies the existence of a polynomial matrix $H(s)$ such that $q V=H R_{2}$, so that $V=G R_{2}$ with $G=\frac{H}{q}$. Conversely, assume that $V=G R_{2}$ for some rational matrix $G(s)$. Since $G(s)$ may be written as $G(s)=\frac{H(s)}{q(s)}$, where $H(s)$ is a polynomial matrix and $q(s)$ is the least common multiple of the denominators of the entries of $G(s)$, it follows that $q V=H R_{2}$, which means that $\operatorname{ker} R_{2} \subset \operatorname{ker} q V$.

\section{Behavioral detectability subspaces}

In the classic geometric approach, detectability subspaces are subspaces $\mathscr{V}_{\mathscr{X}}$ of the state space $\mathscr{X}$ for which there exists a system $\Omega$, with state space $\mathscr{X}$ and state $\widehat{x}$, which is an asymptotic observer for $\mathscr{X} / \mathscr{V}_{\mathscr{X}}$. In other words, denoting the estimation error by $e(t)$, the following conditions should be satisfied [4]:

(i) $\left(e(0) \in \mathscr{V}_{\mathscr{X}}\right) \Rightarrow\left(e(t) \in \mathscr{V}_{\mathscr{X}}, \forall t \geq 0\right)$;

(ii) for all $e(0) \in \mathscr{X}, \lim _{t \rightarrow+\infty}(e(t)) \in \mathscr{V}_{\mathscr{X}}$.

Likewise, behavioral detectability subspaces are defined as behaviors up to which the error dynamics of a suitable observer is stable.

Definition 8: Let $\mathscr{B}_{\left(w_{1}, w_{2}\right)}$ be a behavior and let $\widehat{\mathscr{B}}_{\left(w_{1}, \widehat{w}_{2}\right)}$ be an observer of $w_{2}$ from $w_{1}$ for $\mathscr{B}_{\left(w_{1}, w_{2}\right)}$. Define the corresponding error behavior $\mathscr{B}_{e}$ as in Definition 3. Given a behavior $\mathscr{V}_{e} \subset \mathscr{U}_{e}=\mathscr{U}_{w_{2}}=$ $\mathscr{U}_{\widehat{w}_{2}}$, the observer $\widehat{\mathscr{B}}_{\left(w_{1}, \widehat{w}_{2}\right)}$ is called asymptotic modulo $\mathscr{V}_{e}$ if

$$
\mathscr{V}_{e} \subset \mathscr{B}_{e} \text { and } \mathscr{B}_{e} / \mathscr{V}_{e} \text { is stable. }
$$

Definition 9: Let $\mathscr{B}_{\left(w_{1}, w_{2}\right)}$ be a linear time-invariant differential behavior with measured variable $w_{1}$, and to-be-estimated variable $w_{2}$ in a universe $\mathscr{U}_{w_{2}}$. A behavior $\mathscr{V}_{e} \subset \mathscr{U}_{e}$ is said to be a behavioral detectability subspace of $\mathscr{B}_{\left(w_{1}, w_{2}\right)}$ if $\mathscr{B}_{\left(w_{1}, w_{2}\right)}$ admits an asymptotic observer modulo $\mathscr{V}_{e}$.

The characterization of behavioral detectability subspaces is similar to the one given in Proposition 5 for conditioned invariance, but takes into account the stability requirement of Definition 8, [12].

Proposition 6: Let $\mathscr{B}_{\left(w_{1}, w_{2}\right)}$ be a behavior with hidden behavior $\mathscr{N}_{w_{2}}\left(\mathscr{B}_{\left(w_{1}, w_{2}\right)}\right)=\operatorname{ker} R_{2}$. Then, a behavior $\mathscr{V}_{e} \subset \mathscr{U}_{e}$ described by $\mathscr{V}_{e}=$ $\operatorname{ker} V$ is a behavioral detectability subspace of $\mathscr{B}_{\left(w_{1}, w_{2}\right)}$ if and only if $\exists q \in \mathbb{R}[s]$ stable (i.e., with all zeros in $\mathbb{C}^{-}$) such that $\operatorname{ker} R_{2} \subset \operatorname{ker} q V$.

The proof was omitted in [12] and is therefore presented here.

Proof: "If part:" If a stable polynomial $q(s)$ exists such that $\operatorname{ker} R_{2} \subset \operatorname{ker} q V$, then $\mathscr{E}_{e}:=\operatorname{ker} q V$ contains the hidden behavior $\operatorname{ker} R_{2}$, and is therefore an achievable error behavior. Hence, there exists an observer behavior $\widehat{\mathscr{B}}_{\left(w_{1}, \widehat{w}_{2}\right)}$ for $w_{2}$ from $w_{1}$, such that the corresponding error behavior is $\mathscr{E}_{e}$. Moreover $\mathscr{E}_{e} / \mathscr{V}_{e}=\operatorname{ker} q V / \operatorname{ker} V \simeq$ ker $q I$ is stable, so that $\widehat{\mathscr{B}}_{\left(w_{1}, \widehat{w}_{2}\right)}$ is an asymptotic observer modulo $\mathscr{V}_{e}=\operatorname{ker} V$, and hence $\mathscr{V}_{e}$ is a behavioral detectability subspace.

"Only if part:" If $\mathscr{V}_{e}$ is a behavioral detectability subspace, there exists an achievable error behavior $\mathscr{E}_{e}=\operatorname{ker} E$ such that $\mathscr{V}_{e} \subset \mathscr{E}_{e}$ and $\mathscr{E}_{e} / \mathscr{V}_{e}$ is stable, i.e., there exists an observer $\widehat{\mathscr{B}}_{\left(w_{1}, \widehat{w}_{2}\right)}$ with error behavior $\mathscr{E}_{e}$ which is an asymptotic observer modulo $\mathscr{V}_{e}$. The achievability of the error behavior $\mathscr{E}_{e}$ implies that $\operatorname{ker} R_{2} \subset \operatorname{ker} E$. The inclusions $\operatorname{ker} R_{2} \subset \operatorname{ker} E$ and $\mathscr{V}_{e}=\operatorname{ker} V \subset \mathscr{E}_{e}=\operatorname{ker} E$ show that there exist polynomial matrices $\bar{E}(s)$ and $F(s)$ such that $E=\bar{E} R_{2}, E=F V$, and the fact that $\mathscr{E}_{e} / \mathscr{V}_{e} \simeq \operatorname{ker} F$ is stable shows that $F(s)$ is square and non-singular with stable determinant $q(s):=\operatorname{det} F(s)$.

From $\bar{E} R_{2}=E=F V$, we find $\operatorname{ker} R_{2} \subset \operatorname{ker} F V$ and, clearly,

$\operatorname{ker} F V \subset \operatorname{ker} q V$. Thus, $\operatorname{ker} R_{2} \subset \operatorname{ker} q V$, where $q$ is stable.
Example 3.2: With the previous notation, let $R_{2}(s)=\left[\begin{array}{cc}s^{2} & s \\ -1 & 1 \\ 0 & 1\end{array}\right]$ and $R_{1}(s)=\left[\begin{array}{l}1 \\ 1 \\ 1\end{array}\right]$. Let $V(s)=\left[\begin{array}{ll}s-1 & 1\end{array}\right]$ and $\mathscr{V}_{e}=\operatorname{ker} V\left(\frac{d}{d t}\right)$. Taking $H(s)=\left[\begin{array}{lll}1 & 1 & 0\end{array}\right]$, we have $H(s) R_{2}(s)=(s+1)\left[\begin{array}{ll}s-1 & 1\end{array}\right]=$ $q(s) V(s)$, where $q(s)=s+1$ is stable. Thus, $\mathscr{V}_{e}$ is a behavioral detectability subspace. Indeed, it is easily seen that the observer $H\left(\frac{d}{d t}\right) R_{2}\left(\frac{d}{d t}\right) \widehat{w}_{2}=H\left(\frac{d}{d t}\right) R_{1}\left(\frac{d}{d t}\right) w_{1}$ yields $\left(\frac{d}{d t}+1\right)\left[\frac{d}{d t}-1 \quad 1\right] e=0$, with $e=\widehat{w}_{2}-w_{2}$. Hence, the associated error behavior $\mathscr{E}_{e}=\operatorname{ker}\left(\left(\frac{d}{d t}+\right.\right.$ 1) $\left[\frac{d}{d t}-1 \quad 1\right]$ ) is such that $\mathscr{E}_{e} / \mathscr{V}_{e} \simeq \operatorname{ker}\left(\frac{d}{d t}+1\right)$ is stable

Example 3.3: Consider the state space system described by

$$
\left\{\begin{aligned}
\frac{d}{d t} x_{1} & =2 x_{1} \\
\frac{d}{d t} x_{2} & =3 x_{2} \\
\frac{d}{d t} x_{3} & =-x_{3} \\
y & =x_{1}
\end{aligned}\right.
$$

Defining $R_{2}(s)=\left[\begin{array}{ccc}s-2 & 0 & 0 \\ 0 & s-3 & 0 \\ 0 & 0 & s+1 \\ 1 & 0 & 0\end{array}\right], R_{1}(s)=\left[\begin{array}{l}0 \\ 0 \\ 0 \\ 1\end{array}\right], w_{2}:=\left[\begin{array}{l}x_{1} \\ x_{2} \\ x_{3}\end{array}\right]$, and $w_{1}:=y$, (2) can be written as $R_{2}\left(\frac{d}{d t}\right) w_{2}=R_{1}\left(\frac{d}{d t}\right) w_{1}$. Let $V_{1}(s)=$ $\left[\begin{array}{ccc}s+2 & 0 & 0 \\ 0 & s+1 & 0 \\ 0 & 0 & s+1\end{array}\right]$ and $\mathscr{V}_{1}:=\operatorname{ker} V_{1}\left(\frac{d}{d t}\right)$. The identity $q(s) V_{1}(s)=$ $H(s) R_{2}(s)$, where $H(s)=\left[h_{i j}(s)\right]$ is $3 \times 4$, gives

$$
\begin{aligned}
& q(s)(s+2)=h_{11}(s)(s-2)+h_{14}(s) \\
& q(s)(s+1)=h_{22}(s)(s-3) \\
& q(s)(s+1)=h_{33}(s)(s+1),
\end{aligned}
$$

and it is easy to see that there is no stable polynomial $q(s)$ such that (4) holds. Thus, $\mathscr{V}_{1}$ is not a behavioral detectability subspace.

Let now $V_{2}(s)=\left[\begin{array}{ccc}s+2 & 0 & 0 \\ 0 & s-3 & 0 \\ 0 & 0 & s+1\end{array}\right]$ and $\mathscr{V}_{2}:=\operatorname{ker} V_{2}\left(\frac{d}{d t}\right)$. Then, the equality $q(s) V_{2}(s)=H(s) R_{2}(s)$ is satisfied with $q(s)=1$ and $H(s)=$

$\left[\begin{array}{llll}1 & 0 & 0 & 4 \\ 0 & 1 & 0 & 0 \\ 0 & 0 & 1 & 0\end{array}\right]$, and therefore we may conclude that $\mathscr{V}_{2}$ is a detectability $\left[\begin{array}{llll}0 & 0 & 1 & 0\end{array}\right]$

subspace. Indeed, taking the observer given by equations

$$
H\left(\frac{d}{d t}\right) R_{2}\left(\frac{d}{d t}\right) \widehat{w}_{2}=H\left(\frac{d}{d t}\right) R_{1}\left(\frac{d}{d t}\right) w_{1},
$$

one obtains

$$
\left[\begin{array}{ccc}
\frac{d}{d t}+2 & 0 & 0 \\
0 & \frac{d}{d t}-3 & 0 \\
0 & 0 & \frac{d}{d t}+1
\end{array}\right]\left[\begin{array}{l}
\widehat{x}_{1} \\
\widehat{x}_{2} \\
\widehat{x}_{3}
\end{array}\right]=\left[\begin{array}{l}
4 \\
0 \\
0
\end{array}\right] y
$$

where $\left[\begin{array}{lll}\widehat{x}_{1} & \widehat{x}_{2} & \widehat{x}_{3}\end{array}\right]^{\top}=\widehat{w}_{2}$, and, as before, $y=w_{1}$. From (2) and (6), the behavior $\mathscr{E}_{e}$ of the error $e=\widehat{w}_{2}-w_{2}$ is given by

$$
\left(\frac{d}{d t} I-\left[\begin{array}{ccc}
-2 & 0 & 0 \\
0 & 3 & 0 \\
0 & 0 & -1
\end{array}\right]\right) e=0 .
$$

Therefore $\mathscr{E}_{e}=\mathscr{V}_{2}$ and $\mathscr{E}_{e} / \mathscr{V}_{2}=\{0\}$, which is a stable behavior. $\diamond$

\section{BEHAVIORAL ESTIMATION}

Given a state-space system $\Sigma$ with a measurable output $y$ and a non-measurable output $z$, a classical estimation problem is to try to obtain a estimation of $z$ based on the measurement of $y$, via an 
observer $\Omega$ with input $y$, which produces an estimate $\widehat{z}$ of $z$ in the sense that the estimation error $e(t)=\widehat{z}(t)-z(t)$ is asymptotically zero, independently from the initial states of $\Sigma$ and $\Omega$ [4], [1]. A solution to this problem is given by the classical geometric theory for linear state space systems in terms of detectability subspaces, [4], [1].

Here we consider the following estimation problem.

Behavioral Estimation Problem: Given a behavioral system with behavior $\mathscr{B}_{\left(w, w_{1}, w_{2}\right)}$ described by

$$
\left\{\begin{aligned}
R\left(\frac{d}{d t}\right) w & =R_{1}\left(\frac{d}{d t}\right) w_{1} \\
w_{2} & =K\left(\frac{d}{d t}\right) w
\end{aligned}\right.
$$

where $w_{1}$ is available for measurement and $w_{2}$ and $w$ are not, find an asymptotic estimator for $w_{2}$ from $w_{1}$, i.e., find a behavioral system $\Omega_{\left(w_{1}, \widehat{w}_{2}\right)}$ described by

$$
N\left(\frac{d}{d t}\right) \widehat{w}_{2}=P\left(\frac{d}{d t}\right) w_{1},
$$

such that $\lim _{t \rightarrow+\infty} e_{2}(t)=0$, where $e_{2}(t):=\widehat{w}_{2}(t)-w_{2}(t)$.

The following result gives necessary and sufficient conditions for the solvability of the Behavioral Estimation Problem; its proof is constructive, and provides an asymptotic estimator in case it exists.

Theorem 1: Let $\mathscr{B}_{\left(w, w_{1}, w_{2}\right)}$ be described by (7). The Behavioral Estimation Problem of $w_{2}$ from $w_{1}$ is solvable if and only if $\operatorname{ker} K$ is a behavioral detectability subspace for the estimation of $w$ from $w_{1}$.

Proof: "If part". Let $\operatorname{ker} K$ be a behavioral detectability subspace for the estimation of $w$ from $w_{1}$ in the given system. By Proposition 6 , a stable polynomial $q(s)$ exists such that $\operatorname{ker} R \subset \operatorname{ker} q K$, i.e., there exists a polynomial matrix $T(s)$ such that

$$
q K=T R .
$$

Consider the following estimator for $w$ :

$$
T R \widehat{w}=T R_{1} w_{1}
$$

Since $R w=R_{1} w_{1}$, we obtain $T R w=T R_{1} w_{1}$, which implies that the estimation error $e:=\widehat{w}-w$ for $w$ must satisfy the equation $T R e=$ 0 . However, by (9), this implies $q K e=0$. Letting $\widetilde{e}:=K e$, we find $q \widetilde{e}=0$, and the stability of $q$ implies that $\lim _{t \rightarrow+\infty} \widetilde{e}(t)=0$. Now define an estimate for $w_{2}$ as

$$
\widehat{w}_{2}=K \widehat{w} .
$$

Since, by (8), $w_{2}=K w$, the estimation error $e_{2}=\widehat{w}_{2}-w_{2}$ for $w_{2}$ must satisfy

$$
e_{2}=K e
$$

Thus, by (12), we have $\lim _{t \rightarrow+\infty} e_{2}(t)=\lim _{t \rightarrow+\infty} \widetilde{e}(t)=0$.

Hence, by (10) and (11), the estimate $\widehat{w}_{2}$ for $w_{2}$ is obtained from

$$
\left\{\begin{array}{c}
T R \widehat{w}=T R_{1} w_{1} \\
\widehat{w}_{2}=K \widehat{w}
\end{array} \Leftrightarrow\left[\begin{array}{cc}
T R_{1} & 0 \\
0 & I
\end{array}\right]\left[\begin{array}{l}
w_{1} \\
\widehat{w}_{2}
\end{array}\right]=\left[\begin{array}{c}
T R \\
K
\end{array}\right] \widehat{w} .\right.
$$

In order to obtain a direct relation between $\widehat{w}_{2}$ and $w_{1}$, we eliminate the variable $\widehat{w}$ by pre-multiplying both sides of the previous equation by a minimal left annihilator ${ }^{3}$ (MLA) $\left[\begin{array}{ll}M & -N\end{array}\right]$ of $\left[\begin{array}{c}T R \\ K\end{array}\right]$, see e.g. [15, Cor. 2.38]. This yields the asymptotic estimator for $w_{2}$ from $w_{1}$

$$
N \widehat{w}_{2}=P w_{1} \text { with } P=M T R_{1} .
$$

\footnotetext{
${ }^{3}$ Given two polynomial matrices $L(s)$ and $M(s), L(s)$ is said to be a minimal left annihilator of $M(s)$ if $L(s) M(s)=0$, i.e., $L(s)$ is an annihilator of $M(s)$, and, moreover, every other annihilator of $M(s)$ is a left multiple of $L(s)$.
}

"Only if part". Let $N \widehat{w}_{2}=P w_{1}$ be an asymptotic estimator for $w_{2}$ from $w_{1}$ for system (8). The corresponding estimation error $e_{2}:=$ $\widehat{w}_{2}-w_{2}$ has a behavior determined by the following equations:

$$
\{\begin{array}{rl}
R w & =R_{1} w_{1} \\
w_{2} & =K w \\
N \widehat{w}_{2} & =P w_{1} \\
e_{2} & =\widehat{w}_{2}-w_{2} .
\end{array} \Leftrightarrow\left[\begin{array}{l}
0 \\
0 \\
0 \\
I
\end{array}\right] e_{2}=\underbrace{\left[\begin{array}{cccc}
R & -R_{1} & 0 & 0 \\
K & 0 & -I & 0 \\
0 & P & 0 & -N \\
0 & 0 & -I & I
\end{array}\right]}_{\bar{R}}\left[\begin{array}{c}
w \\
w_{1} \\
w_{2} \\
\widehat{w}_{2}
\end{array}\right]
$$

and pre-multiplying both sides by an MLA $\left[\begin{array}{llll}X & Y & Z & W\end{array}\right]$ of $\bar{R}$, we eliminate all the variables except $e_{2}$, and thus obtain the equation corresponding to the behavior $\mathscr{B}_{e_{2}}$ of $e_{2}$, i.e.,

$$
W e_{2}=0 \text {. }
$$

Since $\left[\begin{array}{llll}X & Y & Z & W\end{array}\right]$ is an annihilator of $\bar{R}$, the following matrix equalities must hold:

$$
\left\{\begin{array} { l } 
{ X R + Y K = 0 } \\
{ - X R _ { 1 } + Z P = 0 } \\
{ - Y - W = 0 } \\
{ - Z N + W = 0 }
\end{array} \Leftrightarrow \left\{\begin{array} { l } 
{ X R - Z N K = 0 } \\
{ X R _ { 1 } - Z P = 0 } \\
{ W = - Y } \\
{ Y = - Z N }
\end{array} \Leftrightarrow \left\{\begin{array}{l}
X R=Z N K \\
X R_{1}=Z P \\
W=-Y \\
Y=-Z N
\end{array}\right.\right.\right.
$$

Therefore, by (14),

$$
W e_{2}=0 \Leftrightarrow-Y e_{2}=0 \Leftrightarrow-Z N e_{2}=0 \Leftrightarrow Z N e_{2}=0,
$$

i.e., $\mathscr{B}_{e_{2}}=\operatorname{ker} Z N$. Since, by assumption, the estimator $N \widehat{w}_{2}=P w_{1}$ is asymptotic, $Z N$ must be a full column rank matrix and moreover all the zeros of $Z N$ must be stable (as $\lim _{t \rightarrow+\infty} e_{2}(t)=0$ ).

We next show that $\operatorname{ker} K$ is a behavioral detectability subspace for the estimation of $w$ from $w_{1}$ in the system $R w=R_{1} w_{1}$. To this end, we show that there exists a stable polynomial $q$ such that $\operatorname{ker} R \subset \operatorname{ker} q K$. Let $U(s)$ be a unimodular matrix such that $U Z N=\left[\begin{array}{l}Q \\ 0\end{array}\right]$, where $Q$ is a square nonsingular polynomial matrix, and partition $U$ as $U=\left[\begin{array}{l}U_{1} \\ U_{2}\end{array}\right]$ where $U_{1}$ has the same number of rows as $Q$. Then, by (15),

$$
X R=Z N K \Rightarrow U_{1} X R=U_{1} Z N K=Q K .
$$

Premultiplying both sides of $U_{1} X R=Q K$ by the adjoint matrix $\widetilde{Q}$ of $Q$ yields $\widetilde{Q} U_{1} X R=\widetilde{Q} Q K=\operatorname{det}(Q) K$. Let $A:=\widetilde{Q} U_{1} X$ and $q:=\operatorname{det}(Q)$. In view of the previous equalities, we have $A R=q K$, so that $\operatorname{ker} R \subset$ $\operatorname{ker} A R=\operatorname{ker} q K$. Finally, notice that $q(s)$ is a stable polynomial since its roots coincide with the zeros of the matrices $Q$ and $Z N$, which are stable. It follows that there exists a stable polynomial $q$ s.t. $\operatorname{ker} R \subset$ $\operatorname{ker} q K$ and hence $\operatorname{ker} K$ is indeed a behavioral detectability subspace for the estimation of $w$ from $w_{1}$ in the system $R w=R_{1} w_{1}$.

Remark 3: Note that the asymptotic estimator for $w$ from $w_{1}$ given by (10) does not impose any additional restrictions on the behavior of $w_{1}$ induced by the original system. Indeed, the $w_{1}$-behavior induced by the estimator (10) is described by the equation:

$$
L T R_{1} w_{1}=0,
$$

where $L(s)$ is an MLA of $T R$. However, from the original description (7) it follows that $R_{1} w_{1}=R w$ which, since $L$ is an MLA of $T R$, implies that $L T R_{1} w_{1}=L T R w=0$. This means that the $w_{1}$ behavior induced by the original system is contained in the $w_{1}$ behavior induced by the estimator (10). Hence, this estimator does not impose additional restrictions to the original $w_{1}$-behavior. Since $w_{1}$ is the only variable shared by the original system and the estimator (10), the estimation of $w$ from $w_{1}$ does not interfere with the original system. The same applies to the proposed subsequent estimation procedure for $w_{2}$. Thus, the estimator constructed in the first part of the proof of Theorem 1 is an acceptor, or a nonintrusive observer. 
Example 4.1: Let $\mathscr{B}_{\left(w, w_{1}, w_{2}\right)}$ be described by (7) with $R(s)=$ $\left[\begin{array}{cc}s^{2} & s \\ -1 & 1 \\ 0 & 1\end{array}\right], R_{1}(s)=\left[\begin{array}{l}1 \\ 1 \\ 1\end{array}\right]$ and $K(s)=\left[\begin{array}{ll}s-1 & 1\end{array}\right]$. Then, $T(s) R(s)=$ $q(s) K(s)$ where $T(s)=\left[\begin{array}{lll}1 & 1 & 0\end{array}\right]$, and $q(s)=s+1$ is a stable polynomial. By Proposition 6 this implies that ker $K$ is a behavioral detectability subspace for the estimation of $w$ from $w_{1}$. Hence, by Theorem 1 and its proof, the Behavioral Estimation Problem of $w_{2}$ from $w_{1}$ is solvable by the estimator that results from eliminating the variable $\widehat{w}$ in

$$
\left\{\begin{aligned}
T R \widehat{w} & =T R_{1} w_{1} \\
\widehat{w}_{2} & =K \widehat{w}
\end{aligned}\right.
$$

i.e., in

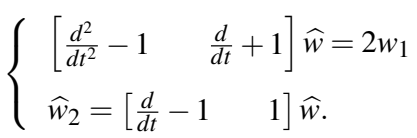

Since $\left[\begin{array}{ll}-1 & s+1\end{array}\right]$ is an MLA of $\left[\begin{array}{c}T R \\ K\end{array}\right]$, after the elimination procedure, one finally obtains the estimator $\left(\frac{d}{d t}+1\right) \widehat{w}_{2}=2 w_{1}$. $\diamond$

\section{BEHAVIORAL ESTIMATION IN THE PRESENCE OF DISTURBANCES}

We now consider the presence of disturbances.

Behavioral Estimation in the presence of Disturbances Problem: Given a behavior $\mathscr{B}_{\left(w, w_{1}, w_{2}, d\right)}$ described by

$$
\left\{\begin{aligned}
R\left(\frac{d}{d t}\right) w & =R_{1}\left(\frac{d}{d t}\right) w_{1}+E\left(\frac{d}{d t}\right) d \\
w_{2} & =K\left(\frac{d}{d t}\right) w
\end{aligned}\right.
$$

where $w_{1}$ is available for measurement, $d$ is an (unknown) disturbance and $w_{2}$ and $w$ are not available for measurement, find a behavioral system $\Omega_{\left(w_{1}, \widehat{w}_{2}\right)}$ governed by

$$
N\left(\frac{d}{d t}\right) \widehat{w}_{2}=P\left(\frac{d}{d t}\right) w_{1},
$$

such that $\lim _{t \rightarrow+\infty} e_{2}(t)=0$, where $e_{2}(t):=\widehat{w}_{2}(t)-$ $w_{2}(t)$, independently from $d$.

This problem can be regarded as a behavioral version of the classical estimation problem with disturbances for state-space systems [4].

Theorem 2: The following conditions are equivalent.

1) The Behavioral Estimation Problem in the presence of Disturbances is solvable.

2) $\operatorname{ker} K$ is a behavioral detectability subspace with respect to the Behavioral Estimation Problem of $w$ from $w_{1}$ in the behavior $\mathscr{B}_{\left(w, w_{1}\right)}:=\left\{\left(w, w_{1}\right): L R w=L R_{1} w_{1}\right\}$, where $L$ is an MLA of $E$.

3) There exists a stable polynomial $q(s)$ such that $\{w: R w \in \operatorname{Im} E\} \subset \operatorname{ker} q K$.

Proof: 1) $\Leftrightarrow 2)$ : The ( $\left.w, w_{1}\right)$-behavior described by (17) can be obtained by eliminating the disturbance $d$ from the description. By the variable elimination principle ([15, Cor. 2.38]), this is achieved by applying an operator $L\left(\frac{d}{d t}\right)$ to both sides of the equation $R w=$ $R_{1} w_{1}+E d$, where $L$ is an MLA of $E$, which yields

$$
\left\{\begin{array}{l}
L R w=L R_{1} w_{1} \\
w_{2}=K w
\end{array}\right.
$$

Now, the original estimation problem with disturbances is equivalent to the estimation of $w$ from $w_{1}$ in (19). This case has been treated in Section IV, and the result follows from Theorem 1.

2) $\Rightarrow 3$ ): If 2) holds, by Proposition 6, a stable polynomial $q(s)$ exists such that $\operatorname{ker} L R \subset \operatorname{ker} q K$. Note that $\operatorname{ker} L=\operatorname{Im} E$ because $L$ is an MLA of $E$, and thus $w \in \operatorname{ker} L R$, i.e., $R w \in \operatorname{ker} L=\operatorname{Im} E$.

3) $\Rightarrow 2):\{w: R w \in \operatorname{Im} E\}=\{w: L R w=0\}=\operatorname{ker} L R$, where $L$ is an MLA of $E$. Now, if condition 3) is satisfied, it follows that $\operatorname{ker} L R \subset$ $\operatorname{ker} q K$ for a suitable stable polynomial $q(s)$. By Proposition 6, this implies that $\operatorname{ker} K$ is a detectability subspace as stated in 2).
Example 5.1: Let $\mathscr{B}_{\left(w, w_{1}, w_{2}, d\right)}$ be described by (17) with $R(s)=$ $\left[\begin{array}{cc}1 & -1 \\ 1 & -1 \\ -s & -1\end{array}\right], R_{1}(s)=\left[\begin{array}{l}1 \\ 1 \\ 1\end{array}\right], E(s)=\left[\begin{array}{c}s-1 \\ s-2 \\ 1\end{array}\right]$ and $K(s)=\left[\begin{array}{ll}s-1 & 2\end{array}\right]$. Consider the Behavioral Estimation Problem of $w$ from $w_{1}$ in the behavior $\mathscr{B}_{\left(w, w_{1}\right)}=\left\{\left(w, w_{1}\right): L R w=L R_{1} w_{1}\right\}$, where $L(s)=\left[\begin{array}{ccc}1 & -1 & -1 \\ s-2 & -(s-1) & 0\end{array}\right]$ is an MLA of $E$. Defining $T(s)=\left[\begin{array}{ll}1 & 1\end{array}\right]$ and the stable polynomial $q(s)=1$, we have that $T(s) L(s) R(s)=q(s) K(s)$, implying that ker $K$ is a behavioral detectability subspace for the estimation of $w$ from $w_{1}$ (in $\mathscr{B}_{\left(w, w_{1}\right)}$ ). Hence, by Theorem 2, the Behavioral Estimation Problem in the presence of Disturbances of $w_{2}$ from $w_{1}$ is solvable. $\diamond$

\section{CONCLUSIONS}

In this paper, we considered the problem of estimation, with and without perturbations, in the behavioral framework. Specifically, given a behavior with partitioned system variable as $\left(w, w_{1}, w_{2}\right)$, where only $w_{1}$ is available for measurement, we defined a Behavioral Estimation Problem and derived necessary and sufficient solvability conditions. Using the elimination principle, we showed that the problem of unknown disturbances affecting the relationship between $w, w_{1}$, and $w_{2}$ can be transformed into the former problem.

\section{REFERENCES}

[1] G. Basile and G. Marro, "Controlled and conditioned invariant subspaces in linear system theory," J. Optim. Theory and Appl., 3(5):306$315,1969$.

[2] J. Willems and C. Commault, "Disturbance decoupling with measurement feedback with stability or pole placement," SIAM J. Contr. Optim., 19(4):490-504, 1981.

[3] J. Willems, "Almost invariant subspaces: an approach to high gain feedback design - Part II: Almost conditionally invariant subspaces," IEEE Trans. Aut. Contr., 5(27):1071-1085, 1982.

[4] H. Trentelman, A. Stoorvogel, and M. Hautus, Control theory for linear systems, Communications and Control Engineering. Springer, 2001.

[5] M. Massoumnia, "A geometric approach to the synthesis of failure detection filters," IEEE Trans. Aut. Contr., 31(9): 839-846, 1986.

[6] M. Hautus, "Strong detectability and observers," Linear Algebra and its Applications, 50:353 - 368, 1983.

[7] M. Hou, A. C. Pugh, and P. C. Muller, "Disturbance decoupled functional observers," IEEE Trans. Aut. Contr., 44(2):382-386, 1999.

[8] Chia-Chi Tsui, "A new design approach to unknown input observers," IEEE Trans. Aut. Contr., 41(3):464-468, 1996.

[9] J. Willems, "Models for dynamics," in Dynamics reported, Vol. 2, ser. Dynam. Report. Ser. Dynam. Systems Appl. Chichester: Wiley, 1989, 2:171-269.

[10] J. Trumpf, H. L. Trentelman, and J. C. Willems, "Internal model principles for observers," IEEE Trans. Aut. Contr., 59(7):1737-1749, 2014.

[11] M. E. Valcher and J. C. Willems, "Observer synthesis in the behavioral approach.” IEEE Trans. Aut. Contr., 44(12):2297-2307, 1999.

[12] R. Pereira and P. Rocha, "Conditioned invariance and detectability subspaces in the behavioral approach," in 20th IFAC World Congress, 50(1):687 - 692, 2017.

[13] - "A remark on conditioned invariance in the behavioral approach," in 2013 Eur. Contr. Conf. (ECC), 301-305, 2013.

[14] J. Polderman and J. Willems, Introduction to Mathematical Systems Theory, A behavioral approach, ser. Texts in Applied Mathematics. New York: Springer-Verlag, 1998, vol. 26.

[15] U. Oberst, "Multidimensional constant linear systems," Acta Appl. Math., 20(1-2):1-175, 1990.

[16] P. Rocha and J. Wood, "Trajectory control and interconnection of 1D and $n \mathrm{D}$ systems," SIAM J. Control Optim., 40(1):107-134, 2001.

[17] J. Wood, "Modules and behaviours in $n \mathrm{D}$ systems theory," Mult. Systems Signal Process., 11(1-2):11-48, 2000.

[18] P. Rocha and J. Wood, "A new perspective on controllability properties for dynamical systems.” Appl. Math. Comput. Sci., 7(4):869-879, 1997. 